\title{
III. Eine nicht zwillingsartige Verwachsung von Natriumchloratkrystallen.
}

\author{
Von \\ W. J. Pope in London. \\ (Hierzu 1 Textfigur.) \\ (Mittheilungen aus dem krystallographischen Laboratorium des »Central \\ Technical Colleges in London, Nr. XV.)
}

Verwachsungen von Krystallen sind entweder Zwillinge, bei welchen eine Drehung des einen Krystallindividuums um eine gemeinsame Zwillingsaxe genügt, um beiden die gleiche Orientirung zu geben, oder aber die Krystallindividuen sind in irgend einer zufälligen Stellung verwachsen, so dass zwischen ihnen keine irgendwie symmetrische Beziehung bezüglich der Orientirung besteht. Der folgende Fall ist von Interesse, weil er augenscheinlich anormal ist in Anbetracht der Art der Verwachsung.

Im Verlaufe der jüngst veröffentlichten Arbeit von Kipping und Pope über Enantiomorphismus (diese Zeitschr. 1898, 30, 472) wurden verschiedene tausend Natriumchloratkrystalle mikroskopisch untersucht. Eine Ernte von Krystallen aus rein wässriger Lösung enthielt drei, deren Aussehen beistehende Figur zeigt. Eine von diesen drei Krystallverwachsungen wurde zur Messung tauglich befunden, und es ergab sich, dass sie nicht nach dem oben erwähnten, gewöhnlich für $Z$ willinge geltenden Gesetze gebildet war; trotzdem waren die zwei Individuen in einer besonderen, symmetrischen

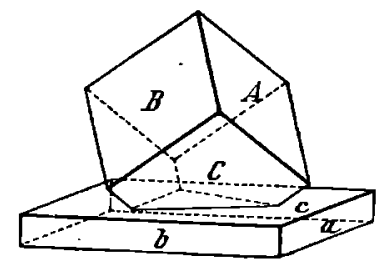
Weise gegen einander orientirt.

Jeder der zwei Krystalle bot alle Flächen der Form $\{100\}$ dar. Der die Basis der Combination bildende Krystall hatte ungefähr ein Quadratcentimeter im Querschnitt und war $1 \mathrm{~mm}$ dick, während der Krystall, wel-. cher auf der ein Quadratcentimeter grossen Basis des ersteren aufsass, einen Würfel von $5-7 \mathrm{~mm}$ Kantenlänge bildete. Die in nachfolgender Tabelle gegebenen Messungen zeigen, dass eine vierzählige Symmetrieaxe des unteren 
16 W. J. Pope. Eine nicht zwillingsartige Verwachsung von Natriumchloratkrystallen.

Krystalles der Richtung nach zusammenfiel mit einer drcizähligen Symmetrieaxe des oberen Krystalles. In der Tabelle beziehen sich rlie grossen Buchstaben auf die Flächen des würfelförmigen Krystalles, deren Indices in Klammern gesetzt sind; die Berechnungen sind unter der Annahme gemacht, dass die dreizählige Symmetrieaxe des einen Krystalles gleichgerichtet mit der vierzähligen des anderen ist, und dass die Flächen $b B C \bar{b}$ in einer Zone liegen.

Winkel: Gemessene Werthe: Berechnete Werthe:

\begin{tabular}{|c|c|c|}
\hline$A B=(100):(010)$ & $89^{0} 56^{\prime}$ & $90^{\circ}$ \\
\hline$B C=(010):(001)$ & 901 & 90 \\
\hline$A C=(100):(001)$ & $90 \quad 5$ & 90 \\
\hline$b B=(\underline{010}):(010)$ & 44. 59 & 4.5 \\
\hline $\bar{b} C=(\underline{0 \bar{\pi} 0}):(001)$ & $45 \quad 3$ & 45 \\
\hline$a A=(\underline{100}):(100)$ & 3518 & $35 \quad 16$ \\
\hline $\bar{a} B=(\underline{T 00}):(010)$ & 6551 & 6554 \\
\hline $\bar{a} C=(\underline{100}):(001)$ & $65 \quad 52$ & $65 \quad 54$ \\
\hline
\end{tabular}

Die nahe Uebereinstimmung zwischen den beobachteten und berechneten Winkeln der Krystallcombination führt zu dem Schlusse, dass die Flächen (111) und $(01 \bar{T})$ des würfelförmigen Krystalles bezüglich parallel sind den Flächen (001) und (010) des tafelförmigen Krystalles.

Beide Individuen waren rechtsdrehend und fest mit einander verwachsen; nach Trennung beider durch Zerbrechen zeigten sie eine unebene Verwachsungsfläche.

Es wäre von Interesse zu wissen, ob derartige Fälle regelmässig orientirter Juxtaposition, bei welcher das allgemeine Gesetz für Zwillinge nicht befolgt ist, häufiger sind. 